\title{
Genetic Analysis for Foliage Yield Attributes in Vegetable Red Amaranth (Amaranthus tricolor L.)
}

\author{
B. S. Asati ${ }^{1 *}$ and M. K. Chandrakar ${ }^{2}$ \\ ${ }^{1}$ Department of Agriculture \& Research Station, Chhuikhadan, Rajnandgaon, \\ Chhattisgarh, India \\ ${ }^{2}$ Pt KLS College of Horticulture and Research Station, Rajnandgaon, India \\ *Corresponding author
}

Keywords

Genetic variability,

Correlation, Path

Coefficient,

Amaranth

Article Info

Accepted:

17 January 2021

Available Online:

10 February 2021

\section{A B S T R A C T}

The present investigation was undertaken to know genetic variability, correlation and path coefficient among yield and its contributing characters in 23 germplasm of red Amaranth collected from different parts of India. The experiment was laid at Pt KLS College of Horticulture and Research Station, Rajnandgaon, Chhattisgarh during 2018-19. The experiment was laid out in randomized complete block design (RCBD) with three replications. High magnitude of genotypic as well as phenotypic coefficient of variations were recorded for traits dry stem, dry plant weight, harvest index, leaf stem ratio and fresh stem weight suggested the substantial improvement on amaranth through selection for these traits. High heritability coupled with high genetic advance was observed for dry stem weight, dry plant weight, leaf stem ratio, fresh stem weight, dry leaf weight, harvest index, leaf area, plant fresh weight, fresh leaf weight indicating that most likely the heritability is due to additive gene effects and selection may be effective. The correlation coefficient analysis revealed that number of leaves per plant and fresh stem weight exhibited the significant positive correlation with leaf yield. Hence, direct selection for these traits may lead to the development of high yielding genotypes of amaranth. The path coefficient analysis revealed that the fresh stem weight, dry leaf weight, petiole length, leaf length, leaf stem ratio, dry stem weight, plant fresh weight, plant height and stem base diameter should be considered in selection criteria for increasing leaf yield.

\section{Introduction}

Amaranth (Chaulai) is one of the important and popular leafy vegetable of India. Vegetable amaranth belongs to the genus
Amaranthus and family Amaranthaceae, composed by approximately 60-70 species. Amaranth (Amaranthus spp.) includes a group of versatile food crops exhibiting high adaptability to new environments, even in the 
presence of different biotic and abiotic stresses (Rana et al., 2007). Red amaranthis a rich source of nutrients it serves as an alternative source of nutrition for rural people in India. In spite of the excellent nutritional qualities of amaranth, not much work has been done on its genetic improvement to increase its foliage yield potential.

Generally the success of any crop improvement program largely depends on the magnitude of genetic variability, heritability, genetic advance, and character association. Genetic variability is important for selection of parents with transgressive segregation (Patro \& Ravisankar, 2004). Heritability estimates provide information on the proportion of phenotypic variance that is due to genetic factors for different traits, but these estimates alone are not a sufficient measure of the level of possible genetic progress. Effective selection can be made when the value of broad sense heritability estimates is considered together with the selection differential or genetic advance (Ibrahim \& Hussein, 2006). Knowledge of correlation between yield and its contributing characters are basic and for most endeavour to find out guidelines for plant selection. Partitioning of total correlation into direct and indirect effect by path coefficient analysis helps in making the selection more effective.

\section{Materials and Methods}

The present investigation was conducted at $\mathrm{Pt}$ KLS College of Horticulture and Research Station, Rajnandgaon, Chhattisgarh during 2018-19. Twenty three diverse genotypes of vegetable amaranth were collected from different agro climatic regions of country (Table 1) and evaluated for various yield and yield attributing traits, correlation and path coefficients were worked out. The single factor experiment was laid out in randomized complete block design (RCBD) with three replications. The seeds are sown in direct field at the distance $15 \mathrm{~cm}$ for row to row and $5 \mathrm{~cm}$ for plant to plant was maintained and the plot size was $1 \mathrm{~m}^{2}$.

Recommended package of practices were adopted for better crop growth. Five competitive plants were selected randomly from each plot to record observation on various characters. Statistical analysis was done by using method suggested by Panse and Sukhatme (1978).

Genotypic and phenotypic coefficients of variation were calculated using the formulae of Burton (1952). The genotypic coefficient of variation $(\mathrm{GCV})$ and phenotypic coefficient of variation $(\mathrm{PCV})$ are categorized as low (less than $10 \%)$, moderate $(10-20 \%)$ and high (more than 20\%) as suggested by Sivasubramanian and Madhavamenon (1973).

The broad sense heritability was estimated for all the characters as the ratio of genotypic variance to total or phenotypic variance (Lush, 1940). The heritability values were classified as low $(<50 \%)$, moderate $(50 \%-70 \%)$ and high (>70\%) as suggested by Robinson (1966). The magnitude of genetic advance as percentage of mean easy categorized as high (>20\%), moderate $(20-10 \%)$ and low $(<10 \%)$ as suggested by Johnson et al., (1955).

The correlation coefficients were determined using variances and co variances to obtain relationship among various characters and their relationship with leaf yield per plant both at genotypic and phenotypic levels by Aljibouri et al., (1958).

The methodology suggested by Wright (1921) and the formula given by Dewey and $\mathrm{Lu}$ (1959) to carry out path coefficient analysis to measure the direct influence of one variable upon the other and to partition the total correlation into direct and indirect effects. 


\section{Results and Discussion}

The analysis of variance of all the characters under study revealed that mean sum of squares due to genotypes was highly significant for all the studied characters. This is an indication of existence of sufficient variability among the genotypes for leaf yield and its component traits. These findings are in general agreement with the findings of Shukla et al., (2005) and Joshi et al., (2011). The extent of variability present in twenty three genotypes of vegetable amaranth was measured in terms of phenotypic coefficient of variation (PCV), genotypic coefficient of variation (GCV), heritability (broad sense) and genetic advance as per cent of mean (GAPM) (Table 2).

Variability refers to the presence of differences among the individuals of a population. Variability is essential for wide adaptability and resistance to biotic and abiotic factors and hence, an insight into the magnitude of genetic variability present in a population is of paramount importance to a plant breeder for starting a judicious breeding programme. The phenotypic and genotypic variances measure the magnitude of variation arising out of difference in phenotypic and genotypic values. The absolute values of phenotypic and genotypic variances cannot be used for comparing the magnitude of variability for different characters, since the mean and units of measurement of the characters may be different. Hence, the coefficients of variation expressed at phenotypic and genotypic levels have been used. The phenotypic coefficient of variation was marginally higher than the corresponding genotypic coefficient of variation indicated the influence of environment in the expression of the character under study (Table 2). High magnitude of genotypic as well as phenotypic coefficient of variations were recorded for traits viz., dry stem weight (32.00 and 33.41 $\%$ ), dry plant weight (28.88 and $31.41 \%$ ), harvest index \% (28.32 and $20.07 \%)$, leaf stem ratio (27.14 and $30.39 \%)$, fresh stem weight (25.60 and $27.81 \%$ ), suggested the substantial improvement on amaranth through selection for these traits. The results are in consonance with Dhangrah et al., (2015) and Gerrano et al., (2015).Moderate GCV and PCV were recorded for dry leaf weight (18.41 and $21.29 \%)$, leaf area (18.38 and $21.91 \%$ ) fresh leaf weight (15.85 and $21.67 \%$ ), plant fresh weight (15.68 and $18.78 \%$ ), petiole length (10.73 and $16.71 \%)$, leaf length (10.60 and $15.23 \%)$ and leaf width (10.28). Suggested existence of considerable variability in the population. Selection for these traits may also be given the importance for improvement programme. Similar findings were also reported earlier by Anuja and Mohideen (2007a) and Venkatesh et al., (2014b).

Heritability estimate provide the information regarding the amount of transmissible genetic variation to total variation and determine genetic improvement and response to selection. In the present investigation high magnitude of heritability was recorded for most of characters. The highest heritability was recorded for the characters dry stem weight $(91.7 \%)$, fresh stem weight $(84.7 \%)$, dry plant weight $(84.6 \%)$, harvest index \% $(83.3 \%)$, Leaf stem ratio $(79.7 \%)$, dry leaf weight $(74.8 \%)$, leaf area $(70.4 \%)$. Similar results reported by Shukla et al., (2006), Anuja and Mohideen (2007a), Das and Kumar (2012). The moderate heritability was observed for Plant fresh weight $(69.8 \%)$ and Fresh leaf weight $(53.5 \%)$. High genetic advance as percentage of mean was observed for dry stem weight (66.66),dry plant weight $(56.66 \%)$, leaf stem ratio $(50.57 \%)$, fresh stem weight (48.68\%), dry leaf weight (34.48 $\%)$, harvest index \% (34.43\%), leaf area $(31.76 \%)$, plant fresh weight $(26.90 \%)$ and fresh leaf weight $(23.84 \%)$. The high value of genetic advance for these traits showed that 
these characters are governed by additive genes and selection will be rewarding for the further improvement of such traits. Heritability estimates along with genetic advance are more useful than the heritability value alone for selecting the best individual. High heritability coupled with high genetic advance was observed for dry stem weight, dry plant weight, leaf stem ratio, fresh stem weight, dry leaf weight, harvest index, leaf area, plant fresh weight, fresh leaf weight indicating that most likely the heritability is due to additive gene effects and selection may be effective. Therefore, selection based on phenotypic performance of these traits would be effective to select desirable plant type. Similar results were also reported Shukla et al., (2005), Anuja and Mohideen (2007a) and Pan et al., (2008).

Association analysis is an important approach in a breeding programme. It gives an idea about relationship among the various characters and determines the component characters, on which selection can be used for genetic improvement in the foliage yield.

The phenotypic correlations were normally of genetic and environmental interaction which provided information about the association between the two characters. Genotypic correlation provided a measure of genetic association between the characters and normally used in selection, while environmental as well as genetic architecture of a genotypes plays a great role in achieving higher yield combined with better quality.

The genotypic and phenotypic correlation for leaf yield and its component in amaranth are presented in Table 3. Plant height showed significant negative correlation with leaf stem ratio, harvest index, number of branches per plant, leaf length, leaf width. Number of branches per plant positively associated with harvest index and also showed significant negative correlation with petiole length. Number of leaves per plant exhibited positive association with fresh stem weight, petiole length, leaf yield, on other hand, it also showed negative correlation with leaf length, leaf stem ratio.

Leaf stem ratio showed significant positive correlation with leaf length, leaf width, leaf area and dry leaf weight at both phenotypic and genotypic levels, whereas, leaf stem ratio exhibited positive association with fresh leaf weight and it also negatively associated with number of leaves per plant at genotypic level only. Plant fresh weight expressed positive correlation with fresh leaf weight and dry leaf weight whereas, fresh leaf weight showed significant positive correlation with dry leaf weight, dry plant weight, leaf stem ratio.

Harvest index showed significant positive correlation with leaf area, leaf stem ratio, number of branches per plant, leaf width showed significant positive correlation with this traits (harvest index), whereas number of leaves per plant showed significant negative correlation with harvest index. The findings clearly indicated that genotypic correlations were of higher magnitude to the corresponding phenotypic ones, thereby establishing strong inherent relationship among the characters studied. The low phenotypic value might be due to appreciable interaction of the genotypes with the environments.

An overall observation of correlation coefficient analysis revealed that number of leaves per plant and fresh stem weight exhibited the significant positive correlation with leaf yield ( $\mathrm{kg} / \mathrm{plot})$. Hence, direct selection for these traits may lead to the development of high yielding genotypes of amaranth. The present findings are in conformity with Navangburuka and Denton (2012), Ahammed et al., (2013) Akaneme and Ani (2013) and Arif et al., (2013). 
Table.1 Treatment Details

\begin{tabular}{|c|c|c|}
\hline S. No. & Treatments/Genotypes & Source \\
\hline 01 & AMAR-01 & Local collection from Hyderabad \\
\hline $\mathbf{0 2}$ & AMAR-02 & Local collection from Delhi \\
\hline 03 & AMAR-03 & Local collection from Delhi \\
\hline 04 & AMAR-04 & Local collection from Raipur \\
\hline 05 & AMAR-05 & Local collection from Jalna \\
\hline 06 & AMAR-06 & Local collection from Faizabad \\
\hline 07 & AMAR-07 & Local collection from Bemetra \\
\hline 08 & AMAR-08 & Local collection from Mungeli \\
\hline 09 & AMAR-09 & Local collection from Raipur \\
\hline 10 & AMAR-10 & Local collection from Kolkata \\
\hline 11 & AMAR-11 & Local collection from Mungeli \\
\hline 12 & AMAR-12 & Local collection from Rajnandgaon \\
\hline 13 & AMAR-13 & Local collection from Hyderabad \\
\hline 14 & AMAR-14 & Local collection from Rajnandgaon \\
\hline 15 & AMAR-15 & Local collection from Bilaspur \\
\hline 16 & AMAR-16 & Local collection from Janjgir \\
\hline 17 & AMAR-17 & Local collection from West Bengal \\
\hline 18 & AMAR-18 & Local collection from Raipur \\
\hline 19 & AMAR-19 & Local collection from Bilaspur \\
\hline 20 & AMAR-20 & Local collection from Raipur \\
\hline 21 & AMAR-21 & Local collection from Raipur \\
\hline 22 & AMAR-22 & Local collection from Rajnandgaon \\
\hline 23 & PusaLalChaulai & Check variety from IARI, New Delhi \\
\hline
\end{tabular}


Table.2 Genetic parameter of variability for leaf yield and its quantitative characters in amaranth

\begin{tabular}{|c|c|c|c|c|c|c|c|c|}
\hline \multirow{2}{*}{ S..No. } & \multirow{2}{*}{ Character } & \multirow{2}{*}{ Mean } & \multicolumn{2}{|c|}{ Range } & \multicolumn{2}{|c|}{$\begin{array}{l}\text { Coefficient of } \\
\text { variation }(\%)\end{array}$} & \multirow{2}{*}{$\begin{array}{l}\text { Heritability } \\
\left(\mathbf{h}^{2} \%\right)\end{array}$} & \multirow{2}{*}{$\begin{array}{c}\text { GA as } \\
\text { percent of } \\
\text { mean }\end{array}$} \\
\hline & & & Min. & Max. & GCV & PCV & & \\
\hline 01 & $\begin{array}{l}\text { Plant height } \\
(\mathrm{cm})\end{array}$ & 15.88 & 12.89 & 17.96 & 7.13 & 12.48 & 32.6 & 8.37 \\
\hline 02 & $\begin{array}{l}\text { No. of branches } \\
\text { per plant }\end{array}$ & 3.11 & 2.67 & 3.53 & 4.59 & 8.83 & 27.0 & 4.82 \\
\hline 03 & $\begin{array}{c}\text { Stem base } \\
\text { diameter }(\mathrm{cm})\end{array}$ & 0.35 & 0.30 & 0.40 & 5.85 & 8.51 & 47.2 & 8.57 \\
\hline 04 & $\begin{array}{l}\text { No. of leaves } \\
\text { per plant }\end{array}$ & 7.46 & 6.40 & 8.13 & 5.32 & 9.36 & 32.3 & 6.30 \\
\hline 05 & $\begin{array}{l}\text { Leaf length } \\
\quad(\mathrm{cm})\end{array}$ & 4.59 & 3.82 & 6.10 & 10.60 & 15.23 & 48.4 & 15.25 \\
\hline 06 & Leaf width $(\mathrm{cm})$ & 3.75 & 3.05 & 4.82 & 10.28 & 14.85 & 47.9 & 14.66 \\
\hline 07 & Leaf area $\left(\mathrm{cm}^{2}\right)$ & 18.70 & 14.13 & 26.86 & 18.38 & 21.91 & 70.4 & 31.76 \\
\hline 08 & $\begin{array}{l}\text { Petiole length } \\
\text { (cm) }\end{array}$ & 2.91 & 2.27 & 3.47 & 10.73 & 16.71 & 41.2 & 14.08 \\
\hline 09 & $\begin{array}{c}\text { Plant fresh } \\
\text { weight }(\mathrm{gm})\end{array}$ & 3.42 & 2.35 & 4.76 & 15.68 & 18.78 & 69.8 & 26.90 \\
\hline 10 & $\begin{array}{c}\text { Fresh leaf } \\
\text { weight (gm) }\end{array}$ & 1.51 & 0.95 & 2.06 & 15.85 & 21.67 & 53.5 & 23.84 \\
\hline 11 & $\begin{array}{c}\text { Fresh stem } \\
\text { weight }(\mathrm{gm})\end{array}$ & 1.52 & 0.95 & 2.37 & 25.60 & 27.81 & 84.7 & 48.68 \\
\hline 12 & $\begin{array}{l}\text { Dry leaf weight } \\
(\mathrm{gm})\end{array}$ & 0.29 & 0.22 & 0.43 & 18.41 & 21.29 & 74.8 & 34.48 \\
\hline 13 & $\begin{array}{c}\text { Dry stem } \\
\text { weight }(\mathrm{gm})\end{array}$ & 0.12 & 0.07 & 0.19 & 32.00 & 33.41 & 91.7 & 66.66 \\
\hline 14 & $\begin{array}{c}\text { Dry plant } \\
\text { weight (gm) }\end{array}$ & 0.30 & 0.21 & 0.49 & 28.88 & 31.41 & 84.6 & 56.66 \\
\hline 15 & $\begin{array}{l}\text { Foliage yield } \\
\text { (kg/plot) }\end{array}$ & 474.68 & 360.67 & 602.87 & 9.64 & 14.34 & 45.2 & 13.35 \\
\hline 16 & Leaf stem ratio & 0.87 & 0.57 & 1.63 & 27.14 & 30.39 & 79.7 & 50.57 \\
\hline 17 & $\begin{array}{c}\text { Harvest } \\
\text { Index }(\%)\end{array}$ & 82.06 & 57.93 & 122.98 & 28.32 & 20.07 & 83.3 & 34.43 \\
\hline
\end{tabular}


Table.3 Genotypic and phenotypic correlation coefficient between leaf yield and its quantitative characters in amaranth

\begin{tabular}{|c|c|c|c|c|c|c|c|c|c|c|c|c|c|c|c|c|c|c|}
\hline \multicolumn{2}{|c|}{ Characters } & $\begin{array}{c}01 . \\
\text { Plant } \\
\text { height } \\
(\mathrm{cm})\end{array}$ & $\begin{array}{c}02 . \\
\text { No. of } \\
\text { Branches } \\
\text { per plant }\end{array}$ & $\begin{array}{c}\mathbf{0 3 .} \\
\text { Stem } \\
\text { base } \\
\text { diameter } \\
(\mathbf{c m})\end{array}$ & $\begin{array}{c}04 . \\
\text { No. of } \\
\text { leaves } \\
\text { Per } \\
\text { plant }\end{array}$ & $\begin{array}{c}05 . \\
\text { Leaf } \\
\text { length } \\
(\mathrm{cm})\end{array}$ & $\begin{array}{c}\text { 06. } \\
\text { Leaf } \\
\text { width } \\
(\mathrm{cm})\end{array}$ & $\begin{array}{l}07 . \\
\text { Leaf } \\
\text { Area } \\
\left(\mathrm{cm}^{2}\right)\end{array}$ & $\begin{array}{c}08 . \\
\text { Petiole } \\
\text { length } \\
(\mathrm{cm})\end{array}$ & $\begin{array}{l}09 . \\
\text { Plant } \\
\text { fresh } \\
\text { weight } \\
(\mathrm{gm})\end{array}$ & $\begin{array}{c}10 . \\
\text { Fresh } \\
\text { Leaf } \\
\text { weight } \\
\text { (gm) }\end{array}$ & $\begin{array}{c}11 . \\
\text { Fresh } \\
\text { stem } \\
\text { weight } \\
\text { (gm) }\end{array}$ & $\begin{array}{c}12 . \\
\text { Dry } \\
\text { Leaf } \\
\text { weight } \\
\text { (gm) }\end{array}$ & $\begin{array}{c}13 . \\
\text { Dry } \\
\text { stem } \\
\text { weight } \\
\text { (gm) }\end{array}$ & $\begin{array}{c}14 . \\
\text { Dry } \\
\text { plant } \\
\text { weight } \\
\text { (gm) }\end{array}$ & $\begin{array}{c}15 . \\
\text { Foliage } \\
\text { yield } \\
\text { (kg/plot) }\end{array}$ & $\begin{array}{c}16 . \\
\text { leaf } \\
\text { stem } \\
\text { ratio }\end{array}$ & $\begin{array}{c}17 . \\
\text { Harvest } \\
\text { Index } \\
(\%)\end{array}$ \\
\hline 01 & $\begin{array}{l}P \\
G\end{array}$ & $\begin{array}{l}1.000 \\
1.000\end{array}$ & $\begin{array}{c}-0.305 \\
-0.659 * *\end{array}$ & $\begin{array}{l}0.282 \\
0.028\end{array}$ & $\begin{array}{l}0.198 \\
0.534\end{array}$ & $\begin{array}{c}-0.061 \\
-0.720 \text { *** }\end{array}$ & $\begin{array}{c}0.067 \\
-0.502 *\end{array}$ & $\begin{array}{l}-0.154 \\
-0.462\end{array}$ & $\begin{array}{l}0.181 \\
0.407\end{array}$ & $\begin{array}{l}0.185 \\
0.389\end{array}$ & $\begin{array}{l}-0.072 \\
-0.048\end{array}$ & $\begin{array}{l}0.325 \\
0.539\end{array}$ & $\begin{array}{l}-0.133 \\
-0.348\end{array}$ & $\begin{array}{l}0.100 \\
0.173\end{array}$ & $\begin{array}{l}0.040 \\
0.127\end{array}$ & $\begin{array}{r}0.069 \\
-0.071\end{array}$ & $\begin{array}{c}-0.512 * \\
-0.828^{* *}\end{array}$ & $\begin{array}{c}-0.528 * \\
-0.843 * *\end{array}$ \\
\hline 02 & $\begin{array}{l}P \\
G\end{array}$ & & $\begin{array}{l}1.000 \\
1.000 \\
\end{array}$ & $\begin{array}{c}-0.175 \\
0.129 \\
\end{array}$ & $\begin{array}{l}-0.164 \\
-0.254\end{array}$ & $\begin{array}{c}-0.017 \\
0.390 \\
\end{array}$ & $\begin{array}{l}0.071 \\
0.340 \\
\end{array}$ & $\begin{array}{l}0.136 \\
0.322 \\
\end{array}$ & $\begin{array}{c}-0.040 \\
-0.491 *\end{array}$ & $\begin{array}{l}-0.206 \\
-0.365\end{array}$ & $\begin{array}{c}0.076 \\
-0.043\end{array}$ & $\begin{array}{c}-0.035 \\
0.178 \\
\end{array}$ & $\begin{array}{c}0.024 \\
-0.238\end{array}$ & $\begin{array}{l}-0.032 \\
-0.015\end{array}$ & $\begin{array}{l}0.024 \\
0.115\end{array}$ & $\begin{array}{c}-0.018 \\
0.150\end{array}$ & $\begin{array}{l}0.095 \\
0.320 \\
\end{array}$ & $\begin{array}{c}0.226 \\
0.675 * *\end{array}$ \\
\hline 03 & $\begin{array}{l}P \\
G\end{array}$ & & & $\begin{array}{l}1.000 \\
1.000\end{array}$ & $\begin{array}{l}0.276 \\
0.516\end{array}$ & $\begin{array}{l}0.342 \\
0.211\end{array}$ & $\begin{array}{l}0.250 \\
0.378\end{array}$ & $\begin{array}{l}0.140 \\
0.199\end{array}$ & $\begin{array}{l}0.270 \\
0.431 \\
\end{array}$ & $\begin{array}{c}0.279 \\
0.483 *\end{array}$ & $\begin{array}{l}0.167 \\
0.242\end{array}$ & $\begin{array}{c}0.460 \\
0.633 * *\end{array}$ & $\begin{array}{l}0.010 \\
0.112\end{array}$ & $\begin{array}{l}0.128 \\
0.205\end{array}$ & $\begin{array}{l}0.233 \\
0.347\end{array}$ & $\begin{array}{l}0.229 \\
0.281\end{array}$ & $\begin{array}{l}0.054 \\
0.227\end{array}$ & $\begin{array}{l}0.202 \\
0.299\end{array}$ \\
\hline 04 & $\begin{array}{l}\mathrm{P} \\
\mathrm{G}\end{array}$ & & & & $\begin{array}{l}1.000 \\
1.000\end{array}$ & $\begin{array}{c}-0.167 \\
-0.506^{*}\end{array}$ & $\begin{array}{l}-0.283 \\
-0.812\end{array}$ & $\begin{array}{l}-0.453 \\
-0.810\end{array}$ & $\begin{array}{c}0.294 \\
0.710^{* * *}\end{array}$ & $\begin{array}{l}0.101 \\
0.302\end{array}$ & $\begin{array}{l}-0.074 \\
-0.189 \\
\end{array}$ & $\begin{array}{c}0.492 * \\
0.852 * *\end{array}$ & $\begin{array}{l}-0.148 \\
-0.200\end{array}$ & $\begin{array}{l}0.210 \\
0.307\end{array}$ & $\begin{array}{l}0.222 \\
0.262\end{array}$ & $\begin{array}{c}0.283 \\
0.490 *\end{array}$ & $\begin{array}{c}-0.226 \\
-0.507 *\end{array}$ & $\begin{array}{c}-0.294 \\
-0.488^{*}\end{array}$ \\
\hline 05 & $\begin{array}{l}P \\
G\end{array}$ & & & & & $\begin{array}{l}1.000 \\
1.000\end{array}$ & $\begin{array}{l}0.711 * * \\
0.904 * *\end{array}$ & $\begin{array}{l}0.811 * * \\
0.936 * *\end{array}$ & $\begin{array}{l}0.227 \\
0.220\end{array}$ & $\begin{array}{l}0.313 \\
0.477\end{array}$ & $\begin{array}{c}0.468 \\
0.868^{* *}\end{array}$ & $\begin{array}{l}-0.058 \\
-0.081\end{array}$ & $\begin{array}{c}0.441 \\
0.757 * *\end{array}$ & $\begin{array}{l}0.075 \\
0.096\end{array}$ & $\begin{array}{l}0.346 \\
0.474\end{array}$ & $\begin{array}{l}0.254 \\
0.416\end{array}$ & $\begin{array}{c}0.586^{*} \\
0.927 * *\end{array}$ & $\begin{array}{c}0.547 * \\
0.895^{* *}\end{array}$ \\
\hline 06 & $\begin{array}{l}P \\
G\end{array}$ & & & & & & $\begin{array}{l}1.000 \\
1.000\end{array}$ & $\begin{array}{l}0.727 * * \\
0.983 * *\end{array}$ & $\begin{array}{r}0.050 \\
-0.011\end{array}$ & $\begin{array}{l}0.201 \\
0.259\end{array}$ & $\begin{array}{l}0.358 \\
0.580\end{array}$ & $\begin{array}{l}-0.116 \\
-0.129\end{array}$ & $\begin{array}{l}0.329 \\
0.459\end{array}$ & $\begin{array}{l}0.103 \\
0.049\end{array}$ & $\begin{array}{l}0.257 \\
0.379\end{array}$ & $\begin{array}{l}0.114 \\
0.077\end{array}$ & $\begin{array}{c}0.493 * \\
0.784 * *\end{array}$ & $\begin{array}{c}0.403 \\
0.800 * *\end{array}$ \\
\hline 07 & $\begin{array}{l}P \\
G\end{array}$ & & & & & & & $\begin{array}{l}1.000 \\
1.000\end{array}$ & $\begin{array}{l}-0.047 \\
-0.096\end{array}$ & $\begin{array}{l}0.234 \\
0.328 \\
\end{array}$ & $\begin{array}{c}0.523^{*} \\
0.753^{* *}\end{array}$ & $\begin{array}{l}-0.226 \\
-0.259\end{array}$ & $\begin{array}{c}0.477 \\
0.640 \text { *** }\end{array}$ & $\begin{array}{l}-0.052 \\
-0.084\end{array}$ & $\begin{array}{l}0.274 \\
0.298\end{array}$ & $\begin{array}{l}0.156 \\
0.128\end{array}$ & $\begin{array}{l}0.614^{* * *} \\
0.795^{* * *}\end{array}$ & $\begin{array}{c}0.605^{*} \\
0.814^{* * *}\end{array}$ \\
\hline 08 & $\begin{array}{l}P \\
G\end{array}$ & & & & & & & & $\begin{array}{l}1.000 \\
1.000\end{array}$ & $\begin{array}{c}0.316 \\
0.503 *\end{array}$ & $\begin{array}{l}0.234 \\
0.456\end{array}$ & $\begin{array}{c}0.328 \\
0.518^{*}\end{array}$ & $\begin{array}{l}0.055 \\
0.090\end{array}$ & $\begin{array}{c}0.445 \\
0.700^{* *}\end{array}$ & $\begin{array}{c}0.363 \\
0.697 * *\end{array}$ & $\begin{array}{l}0.112 \\
0.368\end{array}$ & $\begin{array}{l}-0.085 \\
-0.192\end{array}$ & $\begin{array}{l}-0.120 \\
-0.045\end{array}$ \\
\hline 09 & $\begin{array}{l}P \\
G\end{array}$ & & & & & & & & & $\begin{array}{l}1.000 \\
1.000\end{array}$ & $\begin{array}{c}0.454 \\
0.705^{* *}\end{array}$ & $\begin{array}{l}0.163 \\
0.211\end{array}$ & $\begin{array}{c}0.360 \\
0.488^{*}\end{array}$ & $\begin{array}{l}0.020 \\
0.006\end{array}$ & $\begin{array}{l}0.247 \\
0.379\end{array}$ & $\begin{array}{l}0.150 \\
0.222\end{array}$ & $\begin{array}{l}0.056 \\
0.064\end{array}$ & $\begin{array}{l}-0.042 \\
-0.067\end{array}$ \\
\hline 10 & $\begin{array}{l}\mathrm{P} \\
\mathrm{G}\end{array}$ & & & & & & & & & & $\begin{array}{l}1.000 \\
1.000\end{array}$ & $\begin{array}{l}-0.122 \\
-0.095\end{array}$ & $\begin{array}{c}0.598 * \\
0.852 * *\end{array}$ & $\begin{array}{l}0.175 \\
0.300\end{array}$ & $\begin{array}{c}0.483 * \\
0.690^{* *}\end{array}$ & $\begin{array}{l}0.149 \\
0.225\end{array}$ & $\begin{array}{l}0.374 \\
0.506\end{array}$ & $\begin{array}{l}0.319 \\
0.477\end{array}$ \\
\hline 11 & $\begin{array}{l}P \\
G\end{array}$ & & & & & & & & & & & $\begin{array}{l}1.000 \\
1.000\end{array}$ & $\begin{array}{l}-0.192 \\
-0.202 \\
\end{array}$ & $\begin{array}{l}0.063 \\
0.082\end{array}$ & $\begin{array}{l}0.202 \\
0.240 \\
\end{array}$ & $\begin{array}{c}0.437 \\
0.834^{* *}\end{array}$ & $\begin{array}{l}-0.290 \\
-0.344\end{array}$ & $\begin{array}{l}-0.170 \\
-0.220\end{array}$ \\
\hline 12 & $\begin{array}{l}P \\
G\end{array}$ & & & & & & & & & & & & $\begin{array}{l}1.000 \\
1.000\end{array}$ & $\begin{array}{l}0.053 \\
0.071\end{array}$ & $\begin{array}{c}0.418 \\
0.528^{*}\end{array}$ & $\begin{array}{l}0.161 \\
0.275\end{array}$ & $\begin{array}{c}0.493 * \\
0.612 * *\end{array}$ & $\begin{array}{l}0.252 \\
0.344\end{array}$ \\
\hline 13 & $\begin{array}{l}P \\
G\end{array}$ & & & & & & & & & & & & & $\begin{array}{l}1.000 \\
1.000\end{array}$ & $\begin{array}{l}0.389 \\
0.129\end{array}$ & $\begin{array}{l}-0.073 \\
-0.197\end{array}$ & $\begin{array}{l}-0.130 \\
-0.147\end{array}$ & $\begin{array}{c}-0.009 \\
0.003\end{array}$ \\
\hline 14 & $\begin{array}{l}P \\
G\end{array}$ & & & & & & & & & & & & & & $\begin{array}{l}1.000 \\
1.000\end{array}$ & $\begin{array}{l}0.201 \\
0.254\end{array}$ & $\begin{array}{l}0.252 \\
0.310\end{array}$ & $\begin{array}{l}0.159 \\
0.167 \\
\end{array}$ \\
\hline 15 & $\begin{array}{l}P \\
G\end{array}$ & & & & & & & & & & & & & & & $\begin{array}{l}1.000 \\
1.000\end{array}$ & $\begin{array}{l}0.093 \\
0.125\end{array}$ & $\begin{array}{l}0.156 \\
0.226\end{array}$ \\
\hline 16 & $\begin{array}{l}P \\
G\end{array}$ & & & & & & & & & & & & & & & & $\begin{array}{l}1.000 \\
1.000\end{array}$ & $\begin{array}{l}0.725 * * \\
0.882 * *\end{array}$ \\
\hline 17 & $\begin{array}{l}P \\
G\end{array}$ & & & & & & & & & & & & & & & & & $\begin{array}{l}1.000 \\
1.000\end{array}$ \\
\hline
\end{tabular}


Table.4 Direct and indirect effect of component character on leaf yield in amaranth (Amaranthus tricolor L.)

\begin{tabular}{|c|c|c|c|c|c|c|c|c|c|c|c|c|c|c|c|c|c|}
\hline Characters & $\begin{array}{c}\text { Plant } \\
\text { height } \\
(\mathrm{cm})\end{array}$ & $\begin{array}{c}\text { No. of } \\
\text { Branches } \\
\text { per plant }\end{array}$ & $\begin{array}{c}\text { Stem base } \\
\text { diameter } \\
(\mathrm{cm})\end{array}$ & $\begin{array}{c}\text { No. of } \\
\text { leaves Per } \\
\text { plant }\end{array}$ & $\begin{array}{c}\text { Leaf } \\
\text { length } \\
(\mathrm{cm})\end{array}$ & $\begin{array}{c}\text { Leaf } \\
\text { width } \\
(\mathrm{cm})\end{array}$ & $\begin{array}{l}\text { Leaf } \\
\text { area } \\
\left(\mathrm{cm}^{2}\right)\end{array}$ & $\begin{array}{l}\text { Petiole } \\
\text { length } \\
\text { (cm) }\end{array}$ & $\begin{array}{c}\text { Plant } \\
\text { fresh } \\
\text { weight } \\
\text { (gm) }\end{array}$ & $\begin{array}{c}\text { Fresh } \\
\text { leaf } \\
\text { weight } \\
(\mathrm{gm})\end{array}$ & $\begin{array}{c}\text { Fresh } \\
\text { stem } \\
\text { weight } \\
(\mathrm{gm})\end{array}$ & $\begin{array}{c}\text { Dry leaf } \\
\text { weight } \\
\text { (gm) }\end{array}$ & $\begin{array}{c}\text { Dry stem } \\
\text { weight } \\
\text { (gm) }\end{array}$ & $\begin{array}{c}\text { Dry } \\
\text { plant } \\
\text { weight } \\
(\text { gm) }\end{array}$ & $\begin{array}{l}\text { leaf } \\
\text { stem } \\
\text { ratio }\end{array}$ & $\begin{array}{c}\text { Harvest } \\
\text { Index } \\
(\%)\end{array}$ & $\begin{array}{c}\text { Foliage } \\
\text { yield } \\
\text { (kg/plot) }\end{array}$ \\
\hline Plant height (cm) & $-\underline{0.355}$ & 0.048 & -0.009 & 0.007 & -0.373 & 0.032 & -0.023 & 0.279 & -0.204 & 0.002 & 0.593 & -0.266 & -0.128 & -0.031 & 0.647 & -0.290 & -0.071 \\
\hline $\begin{array}{l}\text { No. of branches per } \\
\text { plant }\end{array}$ & 0.234 & $\underline{-0.073}$ & -0.040 & -0.004 & 0.202 & -0.022 & 0.016 & -0.336 & 0.191 & 0.002 & 0.196 & -0.182 & 0.011 & -0.028 & -0.250 & 0.232 & 0.150 \\
\hline $\begin{array}{c}\text { Stem base diameter } \\
(\mathrm{cm})\end{array}$ & -0.010 & -0.009 & $\underline{-0.306}$ & 0.007 & 0.110 & -0.024 & 0.010 & 0.295 & -0.253 & -0.009 & 0.696 & 0.085 & -0.151 & -0.085 & -0.177 & 0.103 & 0.281 \\
\hline No. of leaves per plant & -0.190 & 0.019 & -0.158 & $\underline{0.014}$ & -0.263 & 0.052 & -0.041 & 0.487 & -0.159 & 0.007 & 0.938 & -0.153 & -.228 & -0.065 & 0.396 & -0.168 & 0.490 \\
\hline Leaf length $(\mathrm{cm})$ & 0.256 & -0.029 & -0.065 & -0.007 & $\underline{0.519}$ & -0.061 & 0.047 & 0.151 & -0.250 & -0.032 & -0.089 & 0.579 & -0.071 & -0.117 & -0.724 & 0.308 & 0.416 \\
\hline Leaf width (cm) & 0.178 & -0.025 & -0.116 & -0.011 & 0.487 & $\underline{-0.065}$ & 0.049 & -0.008 & -0.136 & -0.022 & -0.142 & 0.351 & -0.036 & -0.093 & -0.612 & 0.275 & 0.077 \\
\hline Leaf area $\left(\mathrm{cm}^{2}\right)$ & 0.164 & -0.024 & -0.061 & -0.011 & 0.486 & -0.063 & $\underline{0.050}$ & -0.066 & -0.172 & -0.028 & -0.285 & 0.490 & 0.062 & -0.073 & -0.621 & 0.280 & 0.128 \\
\hline Petiole length $(\mathrm{cm})$ & -0.144 & 0.036 & -0.132 & 0.010 & 0.114 & 0.001 & -0.005 & $\underline{0.686}$ & -0.264 & -0.017 & 0.570 & 0.069 & -0.519 & -0.172 & 0.150 & -0.015 & 0.368 \\
\hline Plant fresh weight (gm) & -0.138 & 0.027 & -0.148 & 0.004 & 0.247 & -0.017 & 0.016 & 0.345 & $\underline{-0.524}$ & -0.026 & 0.232 & 0.374 & -0.004 & -0.093 & -0.050 & -0.023 & 0.222 \\
\hline Fresh leaf weight (gm) & 0.017 & 0.003 & -0.074 & -0.003 & 0.450 & -0.037 & 0.038 & 0.313 & -0.370 & $\underline{-0.037}$ & -0.105 & 0.652 & -0.222 & -0.170 & -0.395 & 0.164 & 0.225 \\
\hline Fresh stem weight (gm) & -0.192 & -0.013 & -0.194 & 0.012 & -0.042 & 0.008 & -0.013 & 0.355 & -0.111 & 0.004 & $\underline{1.100}$ & -0.155 & -0.061 & -0.059 & 0.269 & -0.076 & 0.834 \\
\hline Dry leaf weight (gm) & 0.124 & 0.017 & -0.034 & -0.003 & 0.392 & -0.030 & 0.032 & 0.062 & -0.256 & -0.032 & -0.223 & $\underline{0.766}$ & -0.052 & -0.130 & -0.478 & 0.199 & 0.275 \\
\hline Dry stem weight (gm) & -0.062 & 0.001 & -0.063 & 0.004 & 0.050 & -0.003 & -0.004 & 0.480 & -0.003 & -0.011 & 0.090 & 0.054 & $\underline{-0.741}$ & -0.106 & 0.115 & 0.001 & -197 \\
\hline Dry plant weight (gm) & -0.045 & -0.008 & -0.106 & 0.004 & 0.246 & -0.024 & 0.015 & 0.478 & -0.199 & -0.026 & 0.265 & 0.404 & -0.318 & $\underline{-0.246}$ & -0.242 & 0.057 & 0.254 \\
\hline Leaf stem ratio & 0.294 & -0.023 & -0.069 & -0.007 & 0.481 & -0.051 & 0.040 & -0.132 & -0.034 & -0.019 & -0.379 & 0.469 & 0.109 & -0.076 & $\underline{-0.781}$ & -0.304 & 0.125 \\
\hline Harvest Index(\%) & 0.299 & -0.050 & -0.092 & -0.007 & 0.464 & -0.052 & 0.041 & -0.031 & 0.035 & -0.018 & -0.242 & 0.264 & -0.002 & -0.041 & -0.689 & $\underline{0.344}$ & 0.226 \\
\hline
\end{tabular}


Path coefficient analysis is an important tool for partitioning the correlation coefficients into the direct and indirect effects of independent variables on a dependent variable. With the inclusion of more variables in correlation study, their indirect association becomes more complex. Two characters may show correlation, just because they are correlated with a common third one. In such circumstances, path coefficient analysis provides an effective means of a critical examination of specific forces action to produce a given correlation and measure the relative importance of each factor.

In this analysis, leaf yield was taken as dependent variable and the rest of the characters were considered as undependable variables (Table 4). The path coefficient analysis revealed that fresh stem weight of plant showed the highest positive direct effect (1.100) on fresh stem weight followed by dry leaf weight (0.766), petiole length (0.686), leaf length (0.519), harvest index \% (0.344), leaf area $(0.050)$ and number of leaves per plant (0.014) whereas, the leaf stem ratio $(-0.781)$, dry stem weight $(-0.741)$, plant fresh weight ($0.524)$, plant height $(-0.355)$, stem base diameter (-0.306), number of leaves per plant $(-0.073)$, leaf width $(-0.065)$, fresh leaf weight $(-0.037)$, showed maximum negative direct effects on leaf yield $\mathrm{kg}$ per plot.

Therefore, the higher fresh stem weight and number of leaves per plant should be considered in selection criteria for increasing leaf yield. The present study suggested that more emphasis should be given to selecting genotypes with high fresh stem weight and number of leaves per plant. Directly or indirectly all characters showed positive effect on leaf yield per plant, which is in confirmation to the finding of Sarker et al., (2014) and Venkatesh et al., (2014a). The analysis of variance indicated that the mean sum of square due to genotypes were highly significant for all the studied characters.
Significant mean sum of squares due to leaf yield and attributing characters revealed existence of considerable variability in material studied for improvement of various traits. High magnitude of genotypic as well as phenotypic coefficient of variations were recorded for traits dry stem, dry plant weight, harvest index, leaf stem ratio and fresh stem weight suggested the substantial improvement on amaranth through selection for these traits.

High heritability coupled with high genetic advance was observed for dry stem weight, dry plant weight, leaf stem ratio, fresh stem weight, dry leaf weight, harvest index, leaf area, plant fresh weight, fresh leaf weight indicating that most likely the heritability is due to additive gene effects and selection may be effective.

The correlation coefficient analysis revealed that number of leaves per plant and fresh stem weight exhibited the significant positive correlation with leaf yield. Hence, direct selection for these traits may lead to the development of high yielding genotypes of amaranth. The path coefficient analysis revealed that the fresh stem weight, dry leaf weight, petiole length, leaf length, leaf stem ratio, dry stem weight, plant fresh weight, plant height and stem base diameter should be considered in selection criteria for increasing leaf yield.

\section{Acknowledgement}

We expresses gratitude to Dr. R.N. Ganguli, Dean, Pt. KLS College of Horticulture and Research Station, Rajnandgaon Indira Gandhi Agricultural University, Raipur for extending financial support and providing facility for conducting this research work. We also a thank to Dr.Jitendra Singh, Professor \& Head, Department of Vegetable Science, College of Agriculture, Raipur for their continued encouragement and support throughout the work. 


\section{References}

Al-Jibouri, H.A., Miller, P.A. and Robinson, H.F. $\quad 1958$. Genotypic and environmental variances and covariances in an upland cotton cross of inter-specific origin. Agronomy Journal.50: 632-636.

Ahammed, A. U., Rahman, M. M. and Mian, M. A. K. 2013.Multivariate analysis in stem amaranth (Amaranthus tricolor). Bangladesh J. Pl Bre and Gen, 26(1): 11- 17.

Akaneme, F.I. and Ani, G.O. 2013.Morphological assessment of genetic variability among accessions of Amaranthus hybridus. The Sci. Tech., J. of Sci. and Tech., 2(2): 26-30.

Anuja, S. and Mohideen, M. 2007 a. Variability, heritability and genetic advance studies in amaranthus (Amaranthus spp.). The Asian Journal of Horticulture, 2(1): 63-66.

Arif, M., Jatoi, S.A., Rafique, T. and Ghafoor, A. 2013.Genetic divergence in indigenous spinach genetic resources for agronomic performance and implication of multivariate analyses for future selection criteria. Sci. Tech. and Dev., 32 (1): 7-15.

Burton, G.W. 1952. Quantitative inheritance in grasses.Proc. 6th Int. Gr. Ld. Cong., 1: 277- 283.

Das, A. and Kumar, D. 2012. Genetic evaluation and characterization of jute (Corchorus spp. L) genotypes using DUS parameters. SAARC J. Agri., 10(2): 147-153.

Deway, D.R. and Lu, K.H. 1959. A correlation and path coefficient analysis of components of crested wheat grass and seed production. Agron. J., 5: 515-518.

Dhangrah, V.K., Mandal J. and Bhat, J.S. 2015.Heritable variation and predicted selection response on green yield and its component traits in vegetable amaranth. International Journal of Bio Resource Environment and Agricultural Sciences, 1 (4): 146-153.

Galton, F. 1988. Correlation and their measurements child head from antropometric data. Proc. Roy. Soc., 45:135-145.

Gerrano A.S.,Rensburg W.S.J.V. and Adebola, P.O. 2015.Genetic diversity of amaranthus species in South Africa. South African Journal of Plant and Soil, 32 (1): 39-46.

Ibrahim, M.M. and Hussein, R.M. 2006. Variability, heritability and genetic advance in some genotypes of roselle (Hibiscus sabdariffa L.). World Journal of Agricultural Science, 2 (3): 340-345.

Johnson, H.W., Robinson, H.F. and Comstock, R.E. 1955. Estimates of genetic environmental variability in soybean.Agron. J., 47: 314-318.

Joshi, V., Vijaya, M., Sireesha, K. and Madhavi, P. 2011. Characterization and preliminary evaluation of Vegetable amaranth (Amaranthus spp.) Vegetable Science, 38(2): 239-240.

Lush, J. L. 1940.Intra-sire correlation and regression of offspring on dam as a method of estimation heritability of characteristics. Proc. Amercian Science An. Prod., 301 - 392.

Navangburuka, C.C. and Denton, O.A. 2012. Heritability character association and genetic advance in six agronomic and yield related characters in leaf Corchorus olitorius, International journal of agricultural research, 7(7): 367-375.

Pan, R.S., Singh, A.K., Kumar, S. and Rai, M. 2008. Genetic variation and character association in vegetable amaranth (Amaranthus tricolor).Vegetable Science, 35(1): 81-83.

Panse, V.G. and P.V. Sukhatme, 1978.Statistical methods for 
agricultural workers. ICAR, New Delhi.

Patro T.S.K. and Ravisankar C, 2004.Genetic variability and multivariate analysis in okra [Abelmoschus esculentus (L.) Moench]. Tropical Agriculture Research, 16: 99-113.

Pearson, K. 1904. On the generalized theory of alternative inheritance with special reference to Mendel Law. Phil. Trans. Loy. Soc., A 203: 53-86.

Rana, J.C., Yadav, S.K., Mandal, S. and Yadav, S. 2005. Genetic divergence and interrelationship analysis in grain amaranth (Amaranthus hypochondriacus) germplasm. Indian J. Genet, 65(2): 99-102.

Robinson, H.F., Comstock, R.E. and Harvey, P.H. 1951. Genotypic and phenotypic correlation in corn and their implication in selection. Agron. J., 43: 262-67.

Sarker, U., Islam, T., Rabbani, G. and Oba, S., 2014. Genotypic variability for nutrient, antioxidant, yield and yield contributing traits in vegetable amaranth Journal of Food, Agriculture \& Environment, 12(3): 168-174.

Searle, S. R. 1961. Phenotypic, genotypic and environmental correlations.
Biometrics, 17: 474-480.

Shukla, S., Bhargava, A., Chatterjee, A., Srivastava A. and Singh, S.P. 2006.Genotypic variability in vegetable amaranth (Amaranthus tricolor L.) for foliage yield and its contributing traits over successive cuttings and years. Division of Genetics and Plant Breding, National Botanical Research Institute, RanaPratapMarg, Lucknow-226001, Euphytica, 151: 103-110.

Shukla, S., Bhargava, A., Chatterjee, A., Srivastava, A. and Singh, S.P. 2005. Estimates of genetic variability in vegetable amaranth (A. tricolor) over different cuttings, HORT. SCI. (PRAGUE), 32(2): 60-67.

Sivasubramanian, J. and Madhavamenon, P. 1973. Genotypic and phenotypic variability in rice. Madras Agric. J. 12: 15-16.

Venkatesh, L., Murthy, N., Manjappa and Nehru, S. D. 2014a. Character association and path co-efficient analysis for various traits in grain amaranth (Amaranthus spp.). Asian J. of Bio Sci., 9: 97-100.

Wright, S. 1921. Correlation and causation. J. Agric. Res., 20: 557-585.

\section{How to cite this article:}

Asati, B. S. and Chandrakar, M. K. 2021. Genetic Analysis for Foliage Yield Attributes in Vegetable Red Amaranth (Amaranthus tricolor L.). Int.J.Curr.Microbiol.App.Sci. 10(02): 2143-2153. doi: https://doi.org/10.20546/ijcmas.2021.1002.255 